\title{
Development and Evolution of Cerebral and Cerebellar Cortex
}

\author{
David C. Van Essen Chad J. Donahue Matthew F. Glasser \\ Department of Neuroscience, Washington University School of Medicine, St. Louis, MO, USA
}

\section{Keywords}

Cerebral cortex - Cerebellum - Development - Evolution .

Human · Nonhuman primate $\cdot$ Neuroanatomy Parcellation challenges imposed by the differences in anatomical and functional organization of cerebellar cortex relative to cerebral cortex.

(c) 2018 S. Karger AG, Basel

\begin{abstract}
Cerebral cortex and cerebellar cortex both vary enormously across species in their size and complexity of convolutions. We discuss the development and evolution of cortical structures in terms of anatomy and functional organization. We propose that the distinctive shapes of cerebral and cerebellar cortex can be explained by relatively few developmental processes, notably including mechanical tension along axons and dendrites. Regarding functional organization, we show how maps of myelin content in cerebral cortex are evolutionarily conserved across primates but differ in the proportion of cortex devoted to sensory, cognitive, and other functions. We summarize recent progress and challenges in (i) parcellating cerebral cortex into a mosaic of distinct areas, (ii) distinguishing cortical areas that correspond across species from those that are present in one species but not another, and (iii) using this information along with surfacebased interspecies registration to gain deeper insights into cortical evolution. We also comment on the methodological
\end{abstract}

\section{Introduction}

The cerebral cortex is the dominant structure of the mammalian brain and plays key roles in a remarkably diverse range of behaviors, including perception, volitional movement, cognition, memory, and emotion. In humans, it is vital for capabilities such as language and tool use that make us unique both as a species and as individuals. Its pint-sized partner, the cerebellar cortex, is less well understood functionally but is widely presumed to play a coordinating role in most or all of the above functions [Strick et al., 2009; Buckner et al., 2011]. Figure 1 illustrates the dramatic differences in brain size, neuronal numbers, and complexity of cerebral convolutions for four primate species (human, chimpanzee, macaque, and marmoset) plus the intensively studied mouse. In each species, cerebral and cerebellar cortex, and the subcortical nuclei they surround, are highly interconnected and function as a well-

\section{KARGER}

(C) 2018 S. Karger AG, Basel

E-Mail karger@karger.com

www.karger.com/bbe
David C. Van Essen

Department of Neuroscience, Washington University School of Medicine 660 South Euclid Avenue

St. Louis, MO 63110 (USA)

E-Mail vanessen@wustl.edu 
Fig. 1. Adapted, with permission, from Herculano-Houzel [2009]. Approximate ratios are based on weight differences; ratios for neuronal number differ modestly.

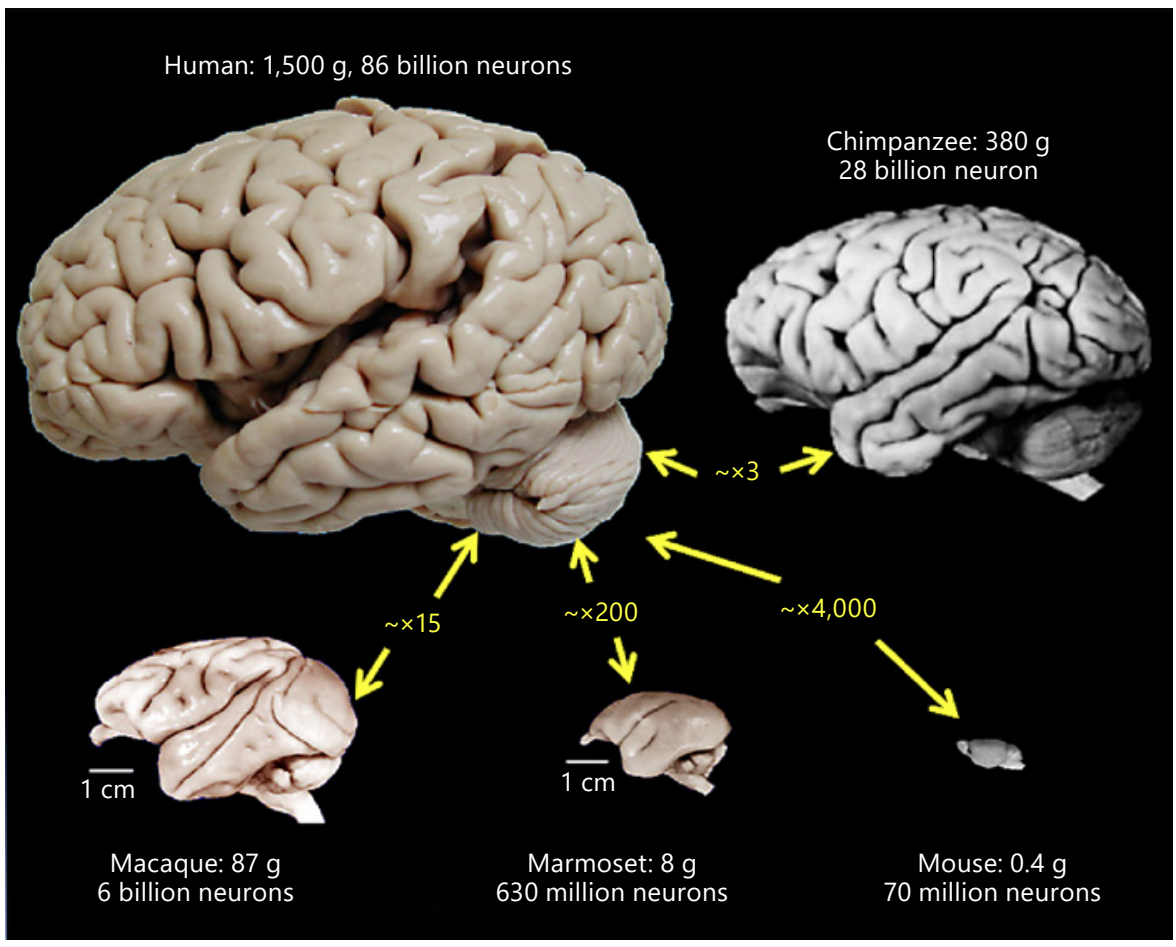

integrated system. This review discusses cerebral and cerebellar cortices from both developmental and evolutionary perspectives, with an emphasis on primates.

\section{Basic Brain Numbers}

The human brain contains $\sim 86$ billion neurons, which are distributed nonuniformly across the cerebellum, cerebral cortex, and subcortical nuclei [Azevedo et al., 2009]. The cerebellum contains $\sim 80 \%$ of the brain's neurons, but it constitutes only $\sim 10 \%$ of brain mass because most of its neurons are tiny granule cells packed into a dense cellular layer within the cerebellar cortical ribbon. In contrast, the cerebral cortex contains only $\sim 20 \%$ of all neurons $(\sim 16$ billion), but it constitutes $\sim 80 \%$ of brain mass as a result of its larger neurons, more abundant neuropil (dendrites, axons, synapses, and glial cells), and the extensive axonal projections that course through the underlying cerebral white matter. The remainder of the brain comprises many diverse subcortical nuclei that, in aggregate, constitute $\sim 8 \%$ of brain mass (including deep subcortical white matter tracts) but contain only $0.8 \%$ of neurons - because these neurons tend to be large and surrounded by neuropil.

Cerebral and cerebellar cortices are both sheet-like structures but otherwise exhibit stark differences. Human cerebral cortex has a total surface area of $1,843 \pm 196 \mathrm{~cm}^{2}$ [both hemispheres; Donahue et al., 2018], about the size of a medium pizza for each hemisphere; it is $2.6 \mathrm{~mm}$ thick on average, but its thickness varies more than twofold across different cortical areas [Glasser et al., 2016b]. Cerebellar cortex is a single sheet, fused along the midline, and it is less than $1 \mathrm{~mm}$ thick (i.e., about one third that of cerebral cortex) (https://msu.edu/ brains/brains/human/coronal/ 2800_cell.html). Its major folds (lamellae) are more regular, akin to those of an accordion, and its total surface area roughly matches that of a single cerebral hemisphere [Van Essen, 2002; Sereno et al., 2015]. In contrast, the subcortical domain includes a collection of nuclei and subnuclei within the thalamus, hypothalamus, basal ganglia, midbrain, and brain stem, with an estimated $\sim 400$ anatomically distinct entities [Forstmann et al., 2016]. Though some nuclei are irregularly shaped (e.g., the long-tailed caudate nucleus), most are blob-like rather than sheet-like.

\section{Morphogenesis - How the Brain Gets in Shape}

Each brain structure attains it size and shape through a tightly choreographed set of morphogenetic events, including neuronal and glial proliferation by cell division, migration from where cells were born to their target 
structure, and the elaboration of axons, dendrites and glial processes. The overall volume of each brain structure can be estimated by its aggregate number of neurons and glial cells (dictated primarily by proliferation but modulated by cell death) multiplied by the average cell volume (including dendrites, axons, synapses, and glial processes). Individual neurons and glial cells have diverse shapes, as their processes extend in characteristic patterns for each tissue and cell type. Dendritic arbors are often biased in orientation (anisotropic), and axonal projections typically extend asymmetrically from the cell body.

In a classic study, Thompson [1917] described how tension and pressure interact with structural anisotropies and asymmetries to determine the shape of many biological structures. If neuronal and glial processes generate mechanical tension, it could be a powerful morphogenetic influence throughout the nervous system [Van Essen, 1997]. In particular, we hypothesize that mechanical tension combined with differential neuronal proliferation plays pivotal roles in answering the following four questions: (i) Why are cerebral and cerebellar cortices sheetlike structures, whereas subcortical nuclei are mostly blob shaped? (ii) Why is cerebral cortex smooth in most smallbrained mammals but convoluted in most large-brained mammals? (iii) What causes the species-specific pattern of convolutions of cerebral cortex? (iv) Why does folding of cerebellar cortex differ so dramatically from that of cerebral cortex?

\section{Are Neurons Tense?}

As mechanical tension is central to our proposed framework, it is important to note that neurons indeed can generate tension. The strongest evidence comes from classic in vitro studies: neurons grown in tissue culture extend neurites whose growth cones attach to the underlying substrate and generate robust mechanical tension [Bray, 1979; Lamoureux et al., 1989; Lamoureux et al., 1992]. In response to external perturbations (e.g., tugging or relaxation), neurites adjust their length by a negative feedback process that tends to maintain a steady tension [Dennerll et al., 1989], much as a fishing line is reeled in or out to regulate tension [Van Essen, 1997]. There is evidence that many axons in subcortical white matter are indeed under tension in brain slices from early postnatal and adult ferrets [Xu et al., 2010], though it is unclear from this study whether axonal tension actually drives cortical folding. Without more fully reviewing a diverse and controversial body of evidence, it suffices for our present purposes to posit that axonal and dendritic tension in vivo is an attractive and highly plausible mecha- nism, though its widespread occurrence in vivo has yet to be rigorously established.

\section{A Role for Tension in Forming Sheet-Like and \\ Blob-Like Structures}

Cerebral cortical neurons proliferate in the ventricular and subventricular zones and migrate outward along radial glial processes to form the cortical plate and later the laminated cortical sheet [Poliakov, 1959]. Shortly after arrival, cortical pyramidal neurons extend a prominent apical dendrite followed by many subsidiary dendrites [Bystron et al., 2008]; the early elaboration of apical dendrites may arise from a diffusible chemoattractant (Sem3A) preferentially released from superficial cortical layers [Polleux et al., 2000]. If these dendrites generate mechanical tension, and if the tufts at apical dendritic tips are anchored by adhesion at synaptic junctions, tension would tend to keep superficial and deep layers close together [Van Essen, 1997]. As cortex expands (by adding neurons, dendrites, synapses, axons, and glial cells), radial tension would keep the cortex thin, and growth would be manifested mainly by tangential expansion along the more compliant dimensions parallel to the surface. In addition, the steady CSF production by the choroid plexus elevates intraventricular hydrostatic pressure [Miller et al., 1987] and would tend to inflate the embryonic cerebral ventricles like a water balloon, thereby enhancing tangential cortical expansion [Van Essen, 1997].

The unique architecture of cerebellar cortex arises from developmental events that differ radically from those in cerebral cortex [Butts et al., 2014]. Early on, Purkinje cell precursors migrate from the ventricular surface, form a monolayer below the cortical surface, and extend dendrites anisotropically towards the pial surface. Precursors of the far more numerous granule cells emerge from the rhombic lip, migrate, continue proliferating in the transient external granule cell layer, and leave a trailing axon while migrating past the Purkinje cells to form the granule cell layer. Radial anisotropies associated with the ascending axons of granule cells, the trellis-like dendritic arbors of Purkinje neurons, and the Bergmann radial glial cells may help keep the cerebellar cortex thin if there is mechanical tension along any or all of these anisotropic components, just as proposed above for cerebral cortex. Additionally, cerebellar cortex has a second, transverse anisotropy involving granule cell axons that branch after ascending, forming parallel fibers that run long distances highly parallel to one another within the superficial cerebellar cortical layer, most commonly along the mediolateral axis. If parallel fibers generate tension, they 
would resist expansion along their long axis, so that tangential expansion would preferentially occur along the more compliant axis orthogonal to the parallel fibers (i.e., along the rostrocaudal axis). Consistent with this hypothesis and reflecting the fact that lamellar folds near the midline tend to run orthogonal to it, the unfolded cerebellar cortex is a highly elongated ribbon after flattening schematically [Sultan and Braitenberg, 1993] or computationally [Van Essen, 2002; Sereno et al., 2015].

In contrast to cortical structures, subcortical neurons typically elaborate dendrites that either radiate quasi-isotropically from the soma or are elongated (e.g., bipolar) but are not aligned consistently relative to their neighbors (see http://neuromorpho.org). If these dendrites also generate mechanical tension, this would tend to keep each subcortical nucleus relatively compact rather than flattening into a sheet. Altogether, mechanical tension along cellular processes in gray matter can account for macroscopic shape differences based on the pattern of anisotropic versus isotropic arborizations.

\section{To Fold or Not?}

Cerebral cortex tends to be convoluted (gyrencephalic) in large brains and smooth (lissencephalic) in small brains (Fig. 1). We hypothesize that cerebral cortex is convoluted to the degree that proliferation of cortical neurons outpaces that of the underlying subcortical nuclei and that this reflects an important correlation between the size of different brain regions and the order of neurogenesis. In an important study, Finlay and Darlington [1995] reported that:

\footnotetext{
"the sizes of brain components, from medulla to forebrain, are highly predictable from absolute brain size by a nonlinear function. The order of neurogenesis was found to be highly conserved across a wide range of mammals and to correlate with the relative enlargement of structures as brain size increases, with disproportionately large growth occurring in late-generated structures."
}

Aspects of this "late equals large" hypothesis have been challenged [see Carlisle et al., 2017 and references therein]. However, for present purposes, it is sufficient to presume that neurogenesis occurs later and more extensively for cerebral neocortex than for various subcortical nuclei and, accordingly, that the ratio of neocortical to subcortical neurons increases disproportionately with brain size. Thus, for small brains, the modest number of cortical neurons forms a sheet whose surface area is just sufficient to surround the subcortical core without major folding. For large brains, the larger number of neocortical neurons and larger cortical surface area exceeds what is needed to envelop the subcortical core, thus predicting the existence of convolutions [Van Essen, 2006], though the specific pattern of folds depends on mechanisms discussed below. In rare situations, large brains may have reduced cortical folding, as in the lissencephalic manatee [Reep and O'Shea, 1990] and also human clinical lissencephaly. However, in these cases, the cortex is unusually thick, resulting in a smaller surface area for a given volume and, hence, fewer convolutions needed to surround the subcortical core, even if cortical volume and neuronal number is in the normal range. Indeed, in humans, thinner regions such as the visual cortex ( $\sim 2 \mathrm{~mm}$ thickness) tend to be folded more tightly than thicker regions like the temporal pole ( $4 \mathrm{~mm}$ thickness).

Cerebellar cortex differs from cerebral cortex in being convoluted in all mammals (and in many nonmammalian vertebrates) [Yopak et al., 2017]. By the logic articulated above, this may reflect the fact that the cerebellar cortex envelops deep cerebellar nuclei whose aggregate volume is small. Hence, even species with small brains have a convoluted cerebellar cortex because its surface area far exceeds that needed to surround the deep nuclei.

\section{How and Where the Cortex Folds}

Much has been written about how the cerebral cortex gets its distinctive folds. Here, we summarize key issues and observations without attempting to be comprehensive. In brief, four main mechanisms have been proposed.

(i) Mechanical tension along the length of axons coursing through the white matter would tend to bring strongly connected regions closer together, thus forming a gyral fold that reduces the wiring length of these connections (e.g., a gyrus between strongly connected areas V1 and V2), whereas sulci would be more likely to form between weakly connected regions (e.g., the central sulcus between weakly connected areas $3 \mathrm{~b}$ and 4 ) as schematized in Figure 2 [Van Essen, 1997]. This hypothesis has great explanatory power, including the ability to explain consistency of folding in regions dominated by large areas with strong and consistent connections and variability of folding in "balkanized" mosaics of smaller areas. Also, tension-based folding would naturally lead to compact wiring ("wiring length minimization"), which is as important for brains [Klyachko and Stevens, 2003; Mota and Herculano-Houzel, 2015] as it is for computer chips. However, there is skepticism in some quarters about tension-based folding [Ronan and Fletcher, 2015]; space limitations here preclude detailed discussion of these criticisms and our specific counterarguments. 


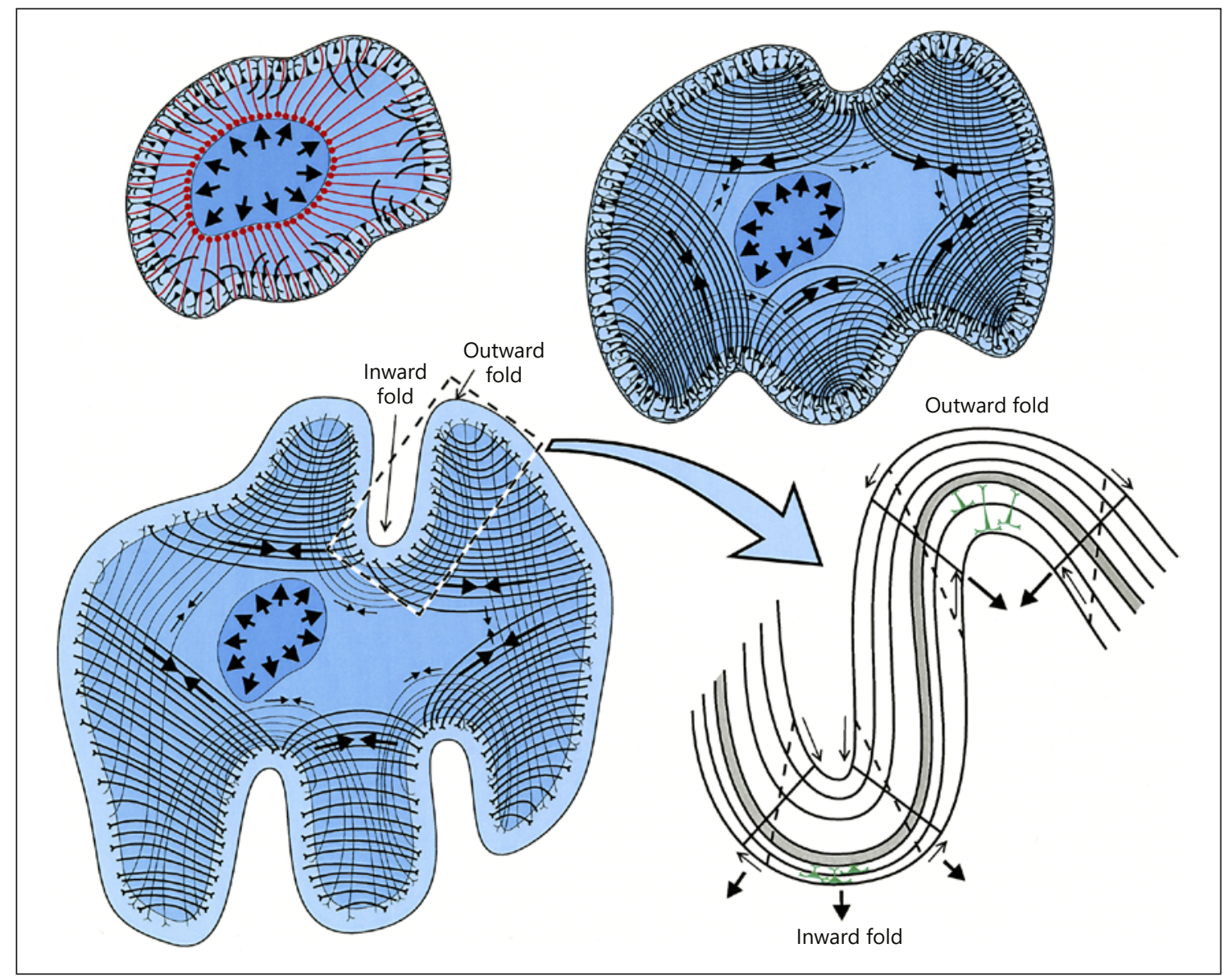

Fig. 2. Schematic drawings of the hypothesized complementary roles of intraventricular pressure and axonal tension in early stages of cortical expansion and gyrification. Reproduced, with permission, from Van Essen [1997].

(ii) Differential regional proliferation in the subventricular zone could produce more neurons in some regions, thereby producing a bulge that then becomes a gyrus, whereas reduced proliferation in nearby subventricular regions would lead to a sulcus or no folding. There is evidence that this may contribute to primary gyri and sulci (early gyrogenesis) in the ferret [Reillo et al., 2011] and plausibly for large early folds in primates such as the sylvian fissure and insula, but it seems highly implausible that this mechanism could account for the complexity of secondary and tertiary folds in highly convoluted brains.

(iii) Mechanical buckling has received considerable attention because of physical and computational models showing convolutions similar to human cortical convolutions from preferential expansion of an initially smooth sheet tethered to an underlying domain that expands more slowly [Tallinen et al., 2014; Tallinen et al., 2016]. However, this model fails to explain the consistencies in major folds relative to large cortical areas (e.g., in the calcarine and central sulci). Stochastic aspects of mechanical buckling may contribute to folding variability in regions where the influence of (i) and (ii) are less strong.

(iv) Differential expansion and/or elasticity of superficial versus deep layers [Richman et al., 1975; Toro and Burnod, 2005] in theory could promote folding by preferential expansion of superficial versus deep layers in regions that become gyri and the reverse for sulci (preferential expansion of deep vs. superficial layers). This would require carefully orchestrated developmental signals for every gyrus and sulcus. In the absence of overt supporting evidence, this mechanism seems highly implausible [but see Ronan and Fletcher, 2015]. Altogether, it seems likely that multiple mechanisms contribute to cortical folding, as others have also suggested [Striedter et al., 2015 and references therein]. These first three mechanisms (axonal tension, differential proliferation, and mechanical buck- 
ling) serve as a particularly plausible combination that can naturally work together.

Cortical folding in the cerebellum is characterized by a set of accordion-like folds that form lobules at the coarsest level, followed by lamellae, and then folia at the finest level. All cerebellar folds tend to run parallel to the long axis of the parallel fibers (i.e., "with the grain") which would allow parallel fibers to slide relative to one another without changing in length. Folding "against the grain" (i.e., orthogonal to their long axis) would be resisted if these axons are under tension, because it would cause stretching of parallel fibers along each outward fold [Van Essen, 1997]. In contrast to cerebral cortex, mechanical tension along long-distance corticocortical axons cannot play a role because no such axons exist (the sole outputs of cerebellar cortex are Purkinje cells projecting to deep nuclei). Tension in axons of ascending inputs might have some influence [Van Essen, 1997], but additional factors not currently understood must be invoked to account for the macroscopic organization of cerebellar folds into its characteristic lobules, lamellae, and folia.

\section{Cortical Evolution}

Cerebral cortical surface areas differ by orders of magnitude across species (even within primates), including an average surface area per hemisphere of $1,843 \mathrm{~cm}^{2}$ for humans, $599 \mathrm{~cm}^{2}$ for chimpanzees, $193 \mathrm{~cm}^{2}$ for macaques, $9.6 \mathrm{~cm}^{2}$ for marmosets, and $0.87 \mathrm{~cm}^{2}$ for mice [Lerch et al., 2008; Chaplin et al., 2013; Donahue et al., 2018]. Given these differences, we explore a few broad questions about cerebral cortex and briefly revisit cerebellar cortex near the end.

What is different about bigger brains, besides sheer size and total number of neurons? For example, to what degree are the individual cortical areas in humans larger than in a chimpanzee or a macaque? Are areal size differences across species reflected in simple scaling relationships? Does human cortex contain entirely new areas not present in chimps or macaques; if so, where are they located? Conversely (given that all primates evolved from a common ancestor), do chimpanzees and/or macaques have novel areas absent in humans? To address questions of this type, it obviously would be ideal if (i) we had accurate parcellations of cortical areas in each species, and (ii) we could readily determine which areas are genuine evolutionary homologues between species (i.e., have a common ancestral origin). Though our current understanding falls well short on both fronts, there is encourag-

Development and Evolution of Cortex ing progress discussed below. First, it is instructive to summarize some "big picture" insights provided by a continuously varying anatomical measure, myelin content, which is indirectly but informatively related to functional organization.

\section{Myelin Maps in Primate Cortex}

Figure 3 shows group average "myelin maps" for human, chimpanzee, and macaque, displayed on inflated left hemisphere surfaces. These maps are based on the ratio of T1-weighted to T2-weighted (T1w/T2w) structural images, computed for each voxel in the gray matter ribbon, mapped to individual surfaces, and then registered to a group average surface. This qualitative, noninvasive measure correlates strongly with postmortem patterns of myelin staining density and is informative about regional aspects of functional specialization [Glasser and Van Essen, 2011; Glasser et al., 2014, 2016b; Nieuwenhuys and Broere, 2017]. Red and orange represent heavily myelinated cortex, which includes "early" areas in somatosensory, motor, auditory, and visual cortex, including the distinctive "MT+" complex of visual areas engaged in motion processing and also retrosplenial cortex. Yellow and green represent moderately myelinated cortex and include areas involved in higher stages of visual, auditory, somatosensory, and motor processing. Blue and indigo represent lightly myelinated cortex that extend over much of frontal, parietal, and lateral temporal regions.

From a functional perspective, there are three striking and interrelated observations: (i) A large portion of the lightly myelinated cortex in humans and macaques is implicated in "higher cognitive" functions, broadly writ, based mainly on neuroimaging in humans, neurophysiology in macaques, and lesion studies in both [Glasser and Rilling, 2008; Ramayya et al., 2010]; presumably, these regions are mainly involved in cognition in chimpanzees, though direct evidence is lacking. (ii) Lightly myelinated regions also have lower neuronal density and larger dendritic arbors [Elston et al., 2009; Glasser et al., 2014], perhaps because these regions in general need more complex cellular machinery to mediate their cognitive functions than do early sensory and motor regions. (iii) The lightly myelinated regions of prefrontal, lateral temporal, and lateral parietal cortices occupy the highest overall fraction of cortex in humans, less in chimpanzees, and still less in macaques; these regions are an even smaller proportion of marmoset cortex [not shown, but see Bock et al., 2009]. As a notable example of interspecies differences, the gap between MT+ and primary auditory cortex (A1) 


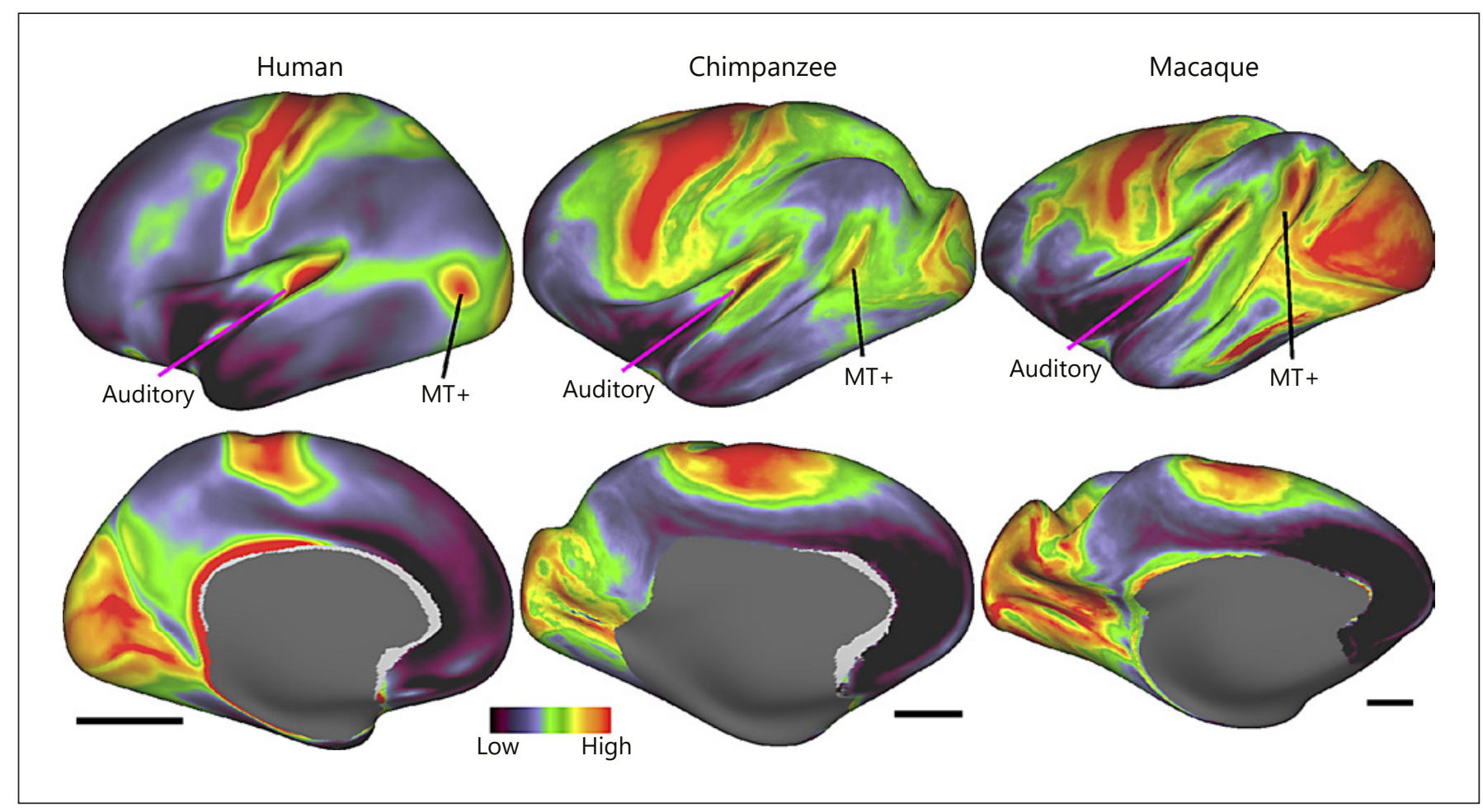

Fig. 3. Group average cortical myelin maps from human, chimpanzee, and macaque. Black bars indicate relative sizes of the group average inflated surfaces for each species. The "MT+" complex refers to heavily myelinated area MT plus the heavily myelinated portions of the neighboring MST complex, also implicated in motion processing (data available at https://balsa.wustl.edu/XPNr).

is a lightly myelinated strip that is narrow in macaques, broader in chimpanzees, and broader still in humans. We revisit these issues below, after considering cortical parcellation and homologies at an areal level.

\section{Cortical Parcellation in General}

Cerebral cortex can be subdivided into a mosaic of areas that can be identified based on differences in function, architecture, connectivity, and topography - the so-called "FACT" approach [Felleman and Van Essen, 1991]. The century-old quest for accurate and reliable parcellations in different species has been reviewed elsewhere [Van Essen and Glasser, 2018]. Here, we summarize highlights for a few species, including mice, marmosets, macaques, and humans, to set the stage for evolutionary comparisons.

\section{Mice}

Mouse neocortex and transitional cortex contains 41 cortical areas based on a recent multimodal analysis [Gămănut et al., 2018]. While unlikely to be a perfect "ground truth" parcellation, this is arguably the closest to a consensus among mammalian species. The primary somatosensory area (SSp) contains seven architectonically distinct subareas representing distinct body regions and illustrates that areas may be internally heterogeneous in some major features yet be properly considered a single well-defined area [Van Essen and Glasser, 2018].

\section{Marmosets}

Recent atlases of marmoset cortex illustrate progress but also challenges in estimating the total number of cortical areas. Figure 4a shows the 3D Paxinos architectonic atlas [Paxinos et al. 2011; Majka et al., 2016] containing 117 areas and displayed on a geometric model of the cortical surface. Figure $4 \mathrm{~b}$ shows the Hashikawa atlas [Hashikawa et al., 2015], also based on the Paxinos atlas. An MRI-based atlas [Liu et al., 2017] contains 54 areas and 106 "subregions." While these atlases agree on many areas, a consensus parcellation is lacking for much of marmoset cortex. A common surface-based atlas would aid in comparing these and other parcellations, as would 


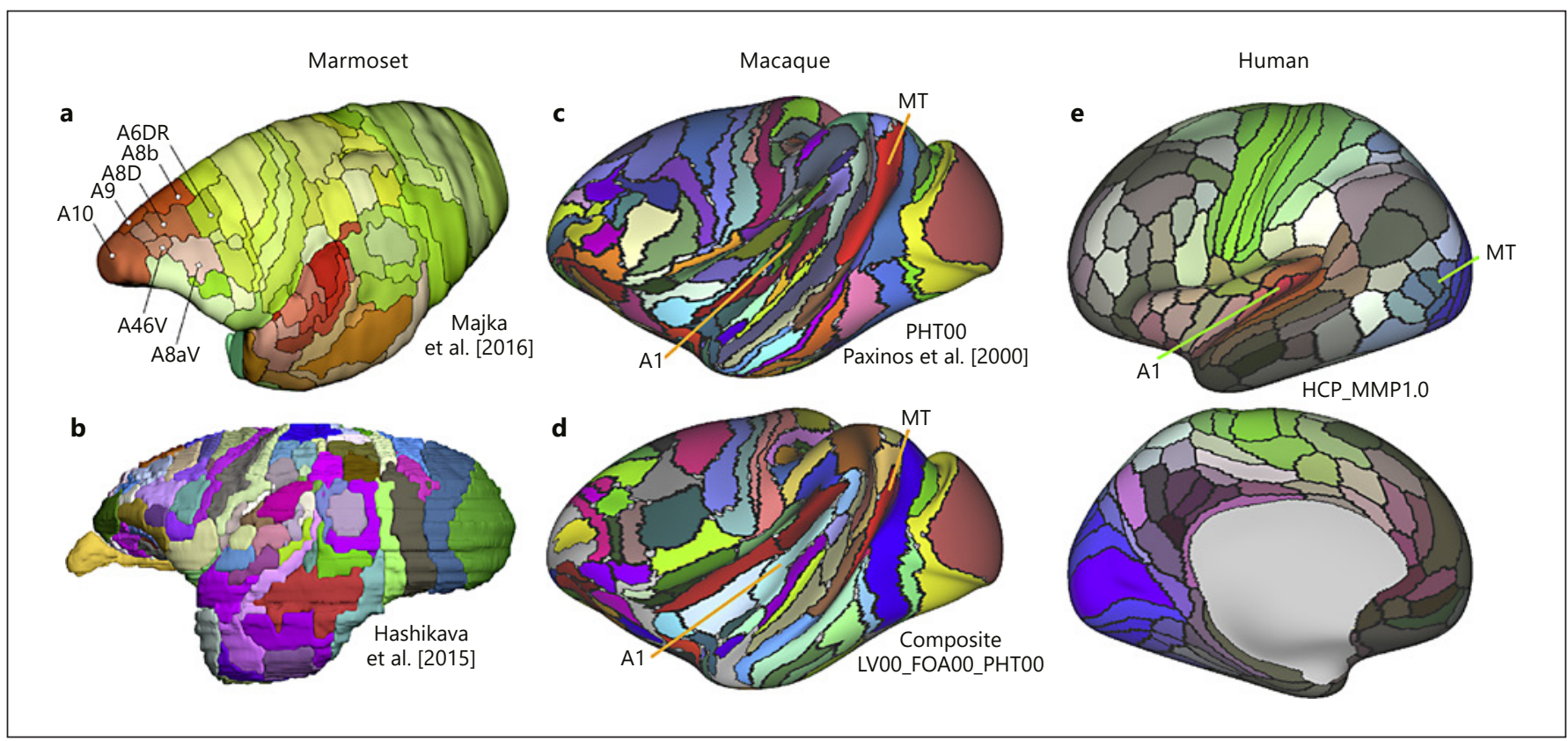

Fig. 4. a, b Marmoset parcellations (a: reproduced with permission from Majka et al. [2016]; b: reproduced with permission from Hashikawa et al. [2015]). c, d Macaque parcellations (c Paxinos et al. [2000]; d Van Essen et al. [2012]). e Human HCP_MMP1.0 parcellation [Glasser et al., 2016a]. Data are available at https://balsa.wustl. edu/8Zqq (c, d) and https://balsa.wustl.edu/KZXj (e).

increased sharing of parcellations and atlases, as done for some of the data in Figures 3 and 4 via the BALSA database (see scene-specific URLs at the end of the figure legends).

\section{Macaques}

Many more parcellations are available for macaques than for marmosets, but the bottom line is similar insofar as there is no consensus parcellation. Mapping parcellations onto a common surface-based atlas, such as the Yerkes19 atlas [Donahue et al., 2016, 2018] facilitates comparisons, as shown in Figure 4c for the 161area Paxinos atlas [Paxinos et al., 2000] and in Figure 4d for a composite 129-area parcellation (extrapolated to $\sim 140$ areas for all of neocortex and transitional cortex) [Van Essen et al., 2012]. Other atlases include a 91-area parcellation [Markov et al., 2014] and the 137-area D99 atlas [Saleem and Logothetis, 2006; Reveley et al., 2017]. Going forward, parcellations based on multiple modalities, acquired in vivo as well as postmortem, may offer the best hope for more closely approaching a consensus.

\section{Humans}

Classical cytoarchitectonic and myeloarchitectonic atlases of human cortex reveal wide divergence across parcellation schemes, especially outside the early sensory and motor areas. Modern parcellations include a growing number based on resting-state functional connectivity. Unfortunately, these generally show major divergence across parcellations; they also differ with consensus architectonic parcellations for early sensory and motor regions and lack bilateral symmetry in regions where it should be expected [Van Essen and Glasser, 2018]. In contrast, the recent "HCP_MMP1.0" multimodal parcellation [Glasser et al., 2016a] stands out from the others because it is based on multimodal imaging data of exceptionally high quality acquired from hundreds of healthy young adults, aligned across individuals using "areal-feature-based" surface registration, and parcellated using an objective gradient-based approach that was overseen by neuroanatomists (Fig. 4e). It contains 180 areas per hemisphere, shows strong bilateral symmetry, and includes a 112-dimensional "feature vector" that characterizes each area. While it is likely to be refined in the future (hence, it is version 1.0), we predict that most of the $\mathrm{HCP}_{-}$ MMP1.0 areas will stand the test of time. 
Some areas in the HCP_MMP1.0 parcellation show internal heterogeneity based on one or another individual modality. This is most notable for the early somatosensory and motor areas, which contain distinct "subareas" that represent specific body subdivisions (e.g., face, upper limb, and lower limb). However, commonalities in architecture and in functional characteristics across the subareas within a larger area (such as somatosensory area $3 \mathrm{a}$ or $3 b)$ support this conceptual distinction between areas and subareas [Van Essen and Glasser, 2018]. On the other hand, some of the higher cognitive domains that are classified as areas in the HCP_MMP1.0 might in the future be reclassified as subareas of a larger area that is topographically organized in a cognitive rather than sensory or motor domain.

\section{Cerebellar Parcellation}

Does the cerebellum have well-defined cortical "areas" identifiable using the "FACT" approaches that have been successful for parcellating cerebral cortex (see above)? In brief, the answer appears to be no (or at least not yet). The functional organization of the cerebellum is complex and may involve organizing principles that differ from cerebral cortex. For example, cerebellar architecture is relatively uniform when examined using conventional Nissl and myelin stains. There is architectonic heterogeneity in the form of parasagittal stripes revealed by immunocytochemical markers [Larouche and Hawkes, 2006], but these are unlikely to reflect areal boundaries. Topographic organization has been mapped for somatosensory regions of the cerebellum. However, rodent cerebellum has a "fractured somatotopy" that differs markedly from cerebral somatotopic maps [Shambes et al., 1978], making it difficult to identify areal boundaries based on a complete body representation. In humans, task-fMRI and functional connectivity analyses reveal two coarse somatomotor maps [Buckner et al., 2011], one dorsomedial and one ventromedial, consistent with neurophysiological maps in the macaque.

Additional clues regarding cerebellar cortical organization come from analyzing its relationship to cerebral cortex that are mediated by indirect, reciprocal pathways. Inputs from the cerebral cortex reach cerebellar cortex via the bottleneck imposed by the much smaller pontine nuclei. Cerebellar outputs (exclusively Purkinje cells) reach cerebral cortex via relays in the deep cerebellar nuclei and thalamus (via the superior cerebellar peduncles). Mapping via transneuronal viral tracers reveal a coarse general topography between cerebral and cerebellar hemispheres [Strick et al., 2009]. In humans, resting-state functional connectivity suggests that cerebellar cortex contains two coarsely homotopic maps of most of the contralateral cerebral cortex, one anterior/dorsal and the other posterior/ventral [Buckner et al., 2011].

Deciphering cerebellar functional organization would be aided by progress in addressing formidable methodological hurdles to applying modern surface-based approaches to analyzing cerebellar cortex. (i) Capturing the shape of cerebellar cortex accurately is difficult because the cortex is thin and tightly folded, and there is little underlying white matter. Segmentation algorithms currently optimized for modeling cerebral cortex will need customization to accurately segment high-resolution cerebellar structural images [Boillat et al., 2018]. (ii) The unfolded cerebellar ribbon is much longer than wide (see above) and is fused along the midline but has prominent lateral hemispheres [Van Essen, 2002; Sereno et al., 2015]. Mapping the cerebellar sheet to a spherical surface while preserving topological relationships entails geometric distortions far more severe than occurs when mapping cerebral cortex to a sphere. This is problematic because current methods for intra- and interspecies registration rely on spheres as the geometric substrates, by deforming one sphere to match corresponding locations on another sphere. If alternate surface-based analysis and visualization methods are developed that can accurately represent cerebellar data, it would open new vistas for the existing high-quality 3- and 7-T data from the Human Connectome Project (HCP) as well as many future studies.

\section{Interspecies Comparisons of Cerebral Cortex}

In this final section, we turn our attention to the evolution of cerebral cortex and briefly discuss two general approaches to comparing cortical organization across species. The widely used "allometric" approach involves measuring in multiple species the size of cortical areas or regions that are presumed to be homologous, then comparing these values across species in absolute terms or in relation to other measures such as total brain size, total cortical size, and/or size of some comparison region of interest. We illustrate this approach with an example that builds on the striking differences in lightly myelinated cortex in humans, chimpanzees, and macaques illustrated in Figure 3. The qualitative impression that prefrontal cortex (PFC) is disproportionately larger in humans relative to nonhuman primates fits with some previous studies but not with others. To address this controversy quantitatively, we analyzed MRI-derived cortical surfaces of many individual humans, chimpanzees, and macaques. Using parcellation-based PFC delineations based on cy- 
toarchitecture and function, we found that the proportion of cortical gray matter occupied by PFC in humans is up to 1.9-fold larger than in macaques and 1.2-fold larger than in chimpanzees [Donahue et al., 2018] relative to total brain size. Thus, we consider human PFC to be disproportionately larger than in other primates. However, it is not exceptionally large, insofar as it is approximately collinear with macaque and chimpanzee on log-log plots relative to total cortical volume.

A powerful complementary approach is to use surfacebased registration to generate a point-to-point mapping between species. In an early example of this approach, a set of 23 landmark contours representing areal boundaries of putative homologous cortical areas was used to register macaque to human cortex [Orban et al., 2004; Van Essen et al., 2005]. This provided evidence for a wide range of relative expansion, from as little as 2 -fold for $\mathrm{V} 1$ and other early sensory areas to more than 30 -fold at the peak of apparent evolutionary hot spots [Van Essen and Dierker, 2007]. However, it is important not to overinterpret these and related findings, as there are significant uncertainties associated with the selection of candidate homologies as well as methodological limitations in the particular landmark-constrained spherical registration algorithm used at the time [see below and Van Essen et al., 2012].

A similar landmark-based approach has been used for interspecies registration between marmosets and capuchins and between marmosets and macaques [Chaplin et al., 2013]. Their marmoset-to-capuchin and marmoset-to-macaque expansion maps show several broad similarities, including hot spots in lateral prefrontal and temporal cortices and "cool spots" in occipital and ventral temporal cortices; these are also roughly similar to the macaque-to-human expansion map noted above [Van Essen and Dierker, 2007]. This suggests that evolutionary scaling relationships have some commonalities across New World as well as Old World primate lineages. However, the differences between each of these pairwise interspecies registrations are also very pronounced. This includes the lateral temporal cortex between MT and A1, which, as already noted, is a narrow gap in the marmoset, much wider in the macaque, and still larger in humans (Fig. 4; see also Fig. 3). Also, in parietal cortex, the expansion maps differ markedly for each pair of species compared. These warrant more detailed analysis using updated maps of plausible homologies across species.

We predict that several factors will accelerate progress in characterizing species differences in cortical organiza- tion and function. (i) More accurate parcellations, particularly in macaques and marmosets, are likely to emerge from projects that acquire high-quality multimodal in vivo neuroimaging data from individual subjects that can be analyzed using "HCP-style" surface-based methods [Glasser et al., 2016b]. (ii) Improved interspecies registration using robust and flexible algorithms, such as multimodal surface matching [Robinson et al., 2017], will more accurately estimate expansion in regions between landmarks and will supplant an older method that tolerated undesirable local nonuniformities [Van Essen et al., 2012]. (iii) Additional algorithmic refinements will enable modeling of areas or regions present in one species but not in another. This may involve a "slit-to-patch" approach in which a region (patch) hypothesized to exist in one species (e.g., various human areas between A1 and MT) emerges from a narrow slit between adjacent areas in the other species (e.g., in the narrow A1-MT gap in the marmoset). (iv) Additional evidence for homologous regions may become available by comparing restingstate networks derived from functional connectivity data using high-quality fMRI data in humans, macaques, and marmosets. Altogether, the prospects are bright for advancing our understanding of cortical organization and evolution through application of cutting-edge approaches.

\section{Acknowledgments}

The study was supported by grants RO1 MH-60974 (D.C.V.E.), NIH 5T32EB01485506 (C.J.D.), and NIH F30 MH097312 (M.F.G.). Human datasets were provided by HCP, WU-Minn Consortium (principal Investigators: David Van Essen and Kamil Ugurbil; 1U54MH091657) funded by the 16 National Institutes of Health (NIH) Institutes and Centers that support the NIH Blueprint for Neuroscience Research; and by the McDonnell Center for Systems Neuroscience at Washington University. Macaque and chimpanzee datasets were provided through support from NIH grant P01AG026423 and the National Center for Research Resources (P51RR165) (superseded by the Office of Research Infrastructure Programs/OD P51OD11132), and by the National Chimpanzee Brain Resource (R24NS092988).

\section{Disclosure Statement}

The authors declare that they have no conflicts of interest pertaining to this paper. 


\section{References}

Azevedo FA, Carvalho LR, Grinberg LT, Farfel JM, Ferretti RE, Leite RE, Jacob Filho W, Lent R, Herculano-Houzel S (2009): Equal numbers of neuronal and nonneuronal cells make the human brain an isometrically scaled-up primate brain. J Comp Neurol 513:532-541.

Bock NA, Kocharyan A, Liu JV, Silva AC (2009): Visualizing the entire cortical myelination pattern in marmosets with magnetic resonance imaging. J Neurosci Methods 185:1522.

Boillat Y, Bazin PL, O'Brien K, MJ Fartaria, Bonnier G, Krueger G, van der Zwaag W, Granziera C (2018): Surface-based characteristics of the cerebellar cortex visualized with ultrahigh field MRI. NeuroImage 172:1-8.

Bray D (1979): Mechanical tension produced by nerve cells in tissue culture. J Cell Sci 37:391410.

Buckner RL, Krienen FM, Castellanos A, Diaz JC, Yeo BT (2011): The organization of the human cerebellum estimated by intrinsic functional connectivity. J Neurophysiol 106:23222345.

Butts T, Green MJ, Wingate RJ (2014): Development of the cerebellum: simple steps to make a "little brain." Development 141:4031-4041.

Bystron I, Blakemore C, Rakic P (2008): Development of the human cerebral cortex: Boulder Committee revisited. Nat Rev Neurosci 9: 110-122.

Carlisle A, Selwood L, Hinds LA, Saunders N, Habgood M, Mardon K, Weisbecker V (2017): Testing hypotheses of developmental constraints on mammalian brain partition evolution, using marsupials. Sci Rep 7:4241.

Chaplin, TA, Yu HH, Soares JG, Gattass T, Rosa MG (2013): A conserved pattern of differential expansion of cortical areas in simian primates. J Neurosci 33:15120-15125.

Dennerll TJ, Lamoureux P, Buxbaum RE, Heidemann SR (1989): The cytomechanics of axonal elongation and retraction. J Cell Biol 109: 3073-3083.

Donahue CJ, Glasser MF, Preuss TM, Rilling JK, Van Essen DC (2018): Quantitative assessment of prefrontal cortex in humans relative to nonhuman primates Proc Natl Acad Sci USA 115:E5183-E51992.

Donahue CJ, Sotiropoulos SN, Jbabdi S, Hernandez-Fernandez M, Behrens TE, Dyrby TB, Coalson T, Kennedy H, Knoblauch K, Van Essen DC, Glasser MF (2016): Using diffusion tractography to predict cortical connection strength and distance: a quantitative comparison with tracers in the monkey. J Neurosci 36:6758-6770.

Elston GN, Oga T, Fujita I (2009): Spinogenesis and pruning scales across functional hierarchies. J Neurosci 29:3271-3275.

Felleman DJ, Van Essen DC (1991): Distributed hierarchical processing in the primate cerebral cortex. Cereb Cortex 1:1-47.
Finlay BL, Darlington RB (1995): Linked regularities in the development and evolution of mammalian brains. Science 268:1578-1584.

Forstmann BU, de Hollander G, van Maanen L, Alkemade A, Keuken MC (2016): Towards a mechanistic understanding of the human subcortex. Nat Rev Neurosci 18:57-65.

Gămănut R, Kennedy H, Toroczkai Z, ErcseyRavasz M, Van Essen DC, Knoblauch K, Burkhalter A (2018): The mouse cortical connectome, characterized by an ultra-dense cortical graph, maintains specificity by distinct connectivity profiles. Neuron 97:698-715.

Glasser MF, Coalson TS, Robinson ED, Hacker CD, Harwell J, Yacoub K, Ugurbil K, Andersson J, Beckmann CF, Jenkinson M, Smith SM, Van Essen DC (2016a): A multi-modal parcellation of human cerebral cortex. Nature 536:171-178.

Glasser MF, Goyal MS, Preuss TM, Raichle ME, Van Essen DC (2014): Trends and properties of human cerebral cortex: correlations with cortical myelin content. NeuroImage 93(pt 2):165-175

Glasser MF, Rilling JK (2008): DTI tractography of the human brain's language pathways. Cereb Cortex 18:2471-2482.

Glasser MF, Smith SM, Marcus DS, Andersson JL, Auerbach EJ, Behrens TE, Coalson TS, Harms MP, Jenkinson M, Moeller S, Robinson EC, Sotiropoulos SN, Xu J, Yacoub E, Ugurbil K, Van Essen DC (2016b): The Human Connectome Project's neuroimaging approach. Nat Neurosci 19:1175-1187.

Glasser MF, Van Essen DC (2011): Mapping human cortical areas in vivo based on myelin content as revealed by T1- and T2-weighted MRI. J Neurosci 31:11597-11616.

Hashikawa T, Nakatomi R, Iriki A (2015): Current models of the marmoset brain. Neurosci Res 93:116-127.

Herculano-Houzel S (2009): The human brain in numbers: a linearly scaled-up primate brain. Front Hum Neurosci 3:31.

Klyachko VA, Stevens CF (2003): Connectivity optimization and the positioning of cortical areas. Proc Nat Acad Sci USA 100:7937-7941.

Lamoureux P, Buxbaum REHeidemann SR (1989): Direct evidence that growth cones pull. Nature 340:159-162.

Lamoureux P, Zheng J, Buxbaum RE, Heidemann SR (1992): A cytomechanical investigation of neurite growth on different culture surfaces. J Cell Biol 118:655-661.

Larouche M, Hawkes R (2006): From clusters to stripes: the developmental origins of adult cerebellar compartmentation. Cerebellum 5: 77-88.

Lerch JP, Carroll JB, Dorr A, Spring S, Evans AC, Hayden MR, Sled JG, Henkelman RM (2008): Cortical thickness measured from MRI in the YAC128 mouse model of Huntington's disease. NeuroImage 41:243-251.
Liu C, Ye FQ, Yen CC, Newman JD, Glen D, Leopold DA, Silva AC (2017): A digital 3D atlas of the marmoset brain based on multi-modal MRI. NeuroImage 169:106-116.

Majka P, Chaplin TA, Yu HH, Tolpygo A, Mitra PP, Wojcik DK, Rosa MG (2016): Towards a comprehensive atlas of cortical connections in a primate brain: mapping tracer injection studies of the common marmoset into a reference digital template. J Comp Neurol 524: 2161-2181.

Markov NT, Ercsey-Ravasz MM, Ribeiro Gomes AR, Lamy C, Magrou L, Vezoli J, Misery P, Falchier A, Quilodran R, Gariel MA, Sallet J, Gamanut R, Huissoud C, Clavagnier S, Giroud P, Sappey-Marinier D, Barone P, Dehay C, Toroczkai Z, Knoblauch K, Van Essen DC Kennedy H (2014): A weighted and directed interareal connectivity matrix for macaque cerebral cortex. Cereb Cortex 24:17-36.

Miller JD, Peeler DF, Pattisapu J, Parent AD (1987): Supratentorial pressures. Part I: differential intracranial pressures. Neurol Res 9: 193-197.

Mota B, Herculano-Houzel S (2015): Cortical folding scales universally with surface area and thickness, not number of neurons. Science 349:74-77.

Nieuwenhuys R, Broere CA (2017): A map of the human neocortex showing the estimated overall myelin content of the individual architectonic areas based on the studies of Adolf Hopf. Brain Struct Funct 222:465-480.

Orban GA, Van Essen D, Vanduffel W (2004): Comparative mapping of higher visual areas in monkeys and humans. Trends Cogn Sci 8: 315-324.

Paxinos G, Huang XF Toga AW (2000): The Rhesus Monkey Brain in Stereotaxic Coordinates. London, Academic Press.

Paxinos G, Watson C, Petrides M, Rosa M, Tokuno H (2011): The Marmoset Brain in Stereotaxic Coordinates, ed 1. London, Academic Press, p 324.

Poliakov GI (1959): Progressive neuron differentiation of the human cerebral cortex in ontogenesis; in Sarkisov SA, Preobrazenskaya SN (eds): Development of the Central Nervous System. Moscow, Medgiz, pp 11-26.

Polleux F, Morrow T, Ghosh A (2000): Semaporin $3 \mathrm{~A}$ is a chemoattractant for cortical apical dendrites. Nature 404:567-573.

Ramayya AG, Glasser MF, Rilling JK (2010): A DTI investigation of neural substrates supporting tool use. Cereb Cortex 20:507-516.

Reep RL, O'Shea TJ (1990): Regional brain morphometry and lissencephaly in the Sirenia. Brain Behav Evol 35:185-194.

Reillo I, de Juan Romero C, Garcia-Cabezas MA, Borrell V (2011): A role for intermediate radial glia in the tangential expansion of the mammalian cerebral cortex. Cereb Cortex 21: 1674-1694. 
Reveley C, Gruslys A, Ye FQ, Glen D, Samaha J, Russ BE, Saad Z, Seth AK, Leopold DA, Saleem KS (2017): Three-dimensional digital template atlas of the macaque brain. Cereb Cortex 27:4463-4477.

Richman DP, Stewart RM, Hutchinson JW, Caviness VS Jr (1975): Mechanical model of brain convolutional development. Science 189:1821.

Robinson EC, Garcia K, Glasser MF, Chen Z, Coalson TS, Makropoulos A, Bozek J, Wright R, Schuh A, Webster M, Hutter J, Price A, Grande LC, Hughes E, Tusor N, Bayly PV, Van Essen DC, Smith SM, Edwards AD, Hajnal J, Jenkinson M, Glocker B, Rueckert D (2017): Multimodal surface matching with higher-order smoothness constraints. NeuroImage 167:453-465.

Ronan L, Fletcher PC (2015): From genes to folds: a review of cortical gyrification theory. Brain Struct Funct 220:2475-2483.

Saleem KS, Logothetis NK (2006): A Combined MRI and Histology Atlas of the Rhesus Monkey Brain. Amsterdam, Academic Press, 2006.

Sereno M, Diedrichsen J, Tachrount MS, De Zeeuw GC (2015): Complete human cerebellar cortex reconstruction and flattening resolving individual folia (abstract). 21st Annu Meet Organization for Human Brain Mapping, Hawaii.
Shambes GM, Gibson JM, Welker W (1978): Fractured somatotopy in granule cell tactile areas of rat cerebellar hemispheres revealed by micromapping. Brain Behav Evol 15:94140.

Strick PL, Dum RP, Fiez JA (2009): Cerebellum and nonmotor function. Annu Rev Neurosci 32:413-434.

Striedter GF, Srinavasan S, Monuki ES (2015): Cortical folding: when, where, how, and why? Ann Rev Neurosci 38:291-307.

Sultan F, Braitenberg V (1993): Shapes and sizes of different mammalian cerebella. A study in quantitative comparative neuroanatomy. J Hirnforsch 34:79-92.

Tallinen T, Chung JY, Biggins JS, Mahadevan L (2014): Gyrification from constrained cortical expansion. Proc Natl Acad Sci USA 111: 12667-12672.

Tallinen T, Chung JY, Rousseau F, Girard N, Lefevre J, Mahadevan L (2016): On the growth and form of cortical convolutions. Nat Phys 12:588-593.

Thompson DW (1917): On Growth and Form. Cambridge, Cambridge University Press, p 793.

Toro R, Burnod Y (2005): A morphogenetic model for the development of cortical convolutions. Cereb Cortex 15:1900-1913.

Van Essen DC (1997): A tension-based theory of morphogenesis and compact wiring in the central nervous system. Nature 385:313318.
Van Essen DC (2002): Surface-based atlases of cerebellar cortex in the human, macaque, and mouse. Ann NY Acad Sci 978:468-479.

Van Essen DC (2006): Cerebral cortical folding patterns in primates: why they vary and what they signify; in Kaas JH (ed): Evolution of Nervous Systems. Oxford, Academic Press, pp 267-276.

Van Essen DC, Dierker DL (2007): Surface-based and probabilistic atlases of primate cerebral cortex. Neuron 56:209-225.

Van Essen DC, Glasser MF (2018): Parcellating cerebral cortex: how invasive animal studies inform non-invasive map-making in humans. Neuron, in preparation.

Van Essen DC, Glasser MF, Dierker DL, Harwell J (2012): Cortical parcellations of the macaque monkey analyzed on surface-based atlases. Cereb Cortex 22:2227-2240.

Van Essen DC, Harwell J, Hanlon D, Dickson J (2005): Surface-based atlases and a database of cortical structure and function; in Koslow SH, Subramaniam S (eds): Databasing the Brain: From Data to Knowledge (Neuroinformatics). Hoboken, Wiley, pp 369-388.

Xu G, Knutsen AK, Dikranian K, Kroenke CD, Bayly PV, Taber LA (2010): Axons pull on the brain, but tension does not drive cortical folding. J Biomech Eng 132:071013.

Yopak KE, Pakan JMP, Wylie D (2017): The cerebellum of nonmammalian vertebrates; in Kaas JH (ed): Evolution of Nervous Systems, ed 2. Oxford, Academic Press, pp 373-386. 